\title{
PARP Inhibitors in Cancer Diagnosis and Therapy
}

Chung Ying Chan ${ }^{1}$, Kel Vin Tan ${ }^{2}$, and Bart Cornelissen ${ }^{1}$

Authors' Affiliations: ${ }^{1}$ MRC Oxford Institute for Radiation Oncology, Department of Oncology, University of Oxford, Oxford, UK and ${ }^{2}$ Department of Diagnostic Radiology, LKS Faculty of Medicine, The University of Hong Kong, Hong Kong

Running Title: PARP imaging and Therapy

Financial Support: This work was supported by Pancreatic Cancer U.K.; CRUK through the Oxford Institute for Radiation Oncology (for BC), and in part by Seed Fund for Basic Research of University of Hong Kong (for KVT and CYC). BC is a paid consultant for Theragnostics Ltd.

Disclosure of Potential Conflicts of Interest: All authors have no potential conflicts of interest to be disclosed.

\section{Corresponding Author:}

Dr Bart Cornelissen, Associate Professor

University of Oxford

MRC Oxford Institute for Radiation Oncology

Department of Oncology

Old Road Campus Research Building

Off Roosevelt Drive

Oxford OX3 7L

United Kingdom

bart.cornelissen@oncology.ox.ac.uk 
Abbreviations

ATK

ATM

BCL2

CI

DLBCL

HER2

$\mathrm{LMO} 2$

MYCN

PIN1

PTEN

R-CHOP

Ataxia Telangiectasia and Rad3-related

Ataxia Telangiectasia Mutated

B-Cell Lymphoma 2

Confidence Interval

Diffuse large B cell lymphomas

Human epidermal growth factor receptor 2

LIM Domain only 2

MYC oncogene (neuroblastoma)

Peptidylprolyl cis/trans Isomerase, NIMA-

Interacting 1

Phosphatase and tensin homolog

Rituximab-Cyclophosphamide-

Hydroxydaunorubicin-Oncovin-Prednisone 


\begin{abstract}
Targeting of poly(ADP-ribose)polymerase (PARP) enzymes has emerged as an effective therapeutic strategy to selectively target cancer cells with deficiencies in homologous recombination (HR) signaling. Currently used to treat $B R C A$-mutated cancers, PARP inhibitors (PARPi) have demonstrated improved outcome in various cancer types as single agents. Ongoing efforts have seen the exploitation of PARPi combination therapies, boosting patient responses as a result of drug synergisms. Despite great successes using PARPi therapy, selecting those patients who will benefit from single agent or combination therapy remains one of the major challenges. Numerous reports have demonstrated that the presence of a $B R C A$ mutation does not always result in synthetic lethality with PARPi therapy in treatment-naïve tumors. Cancer cells can also develop resistance to PARPi therapy. Hence, combination therapy may significantly affect the treatment outcomes. In this review, we discuss the development and utilization of PARPi in different cancer types from preclinical models to clinical trials, provide a current overview of the potential uses of PARP imaging agents in cancer therapy, and discuss the use of radiolabeled PARPi as radionuclide therapies.
\end{abstract}




\section{Introduction}

Targeting DNA damage repair (DDR) signaling is a fast-expanding field for cancer therapy. DNA damage causes genomic instability in cells that require DDR for rescue. DDR signaling triggers activation of repair protein transcription, hence leading to overexpression of the related repair protein and activation of mechanisms for DNA repair (1). Poly(ADP-ribose)polymerase (PARP) is an essential and abundant DNA repair protein, which plays an important role in regulation of various DDR pathways, including Base Excision Repair (BER), Homologous Recombination (HR), classical and alternative Non-Homologous End Joining (NHEJ), Nucleotide Excision Repair (NER), microhomology-mediated end-joining (MMEJ), maintenance of replication fork stability, and Mismatch Repair (MMR) (2). In addition, PARP has been found overexpressed in various types of cancers, such as breast, ovarian and oral cancers than their normal surrounding healthy tissues, making inhibition of PARP activity a promising strategy for cancer therapeutics by disrupting PARP functions, thereby impairing DDR pathways of cancer cells. PARP inhibition for the treatment of breast or ovarian cancers with $B R A C 1 / 2$ mutations (deficiencies in the HR) is now a well-established approach. A PARP inhibitor (PARPi) is utilized to impair the process of single-strand break (SSB) repair, thereby converting SSB into doublestrand breaks (DBSs) by stalling replication folk (1) or accelerating fork elongation (3), which requiring HR for repair during S-phase (4). Therefore, if HR is defective for example, resulting from mutations in HR genes such as BRCA1 and 2, DSBs cannot be repaired, and the cell dies, a concept known as synthetic lethality. Over the past decade, four PARPi (olaparib, rucaparib, niraparib, talazoparib) have been FDAapproved for clinical use as single agents, with veliparib expected to receive approval following promising phase 3 trial results (BROCADE3; NCT02163694). The ability 
of PARPi to impair DDR processes led to their employment in combination with chemo- and radiotherapy. Increasing attention is also being drawn to radiolabeled versions of PARPi that possibly allow direct visualization of PARP expression, quantification of PARPi biodistribution and tumor delivery, as well as target engagement, thereby potentially improve stratification of patients and enable the monitoring of treatment progress. Additionally, radiolabeled PARPi-based radionuclide therapy agents are being proposed as therapies for PARP-expressing cancers. Here, we present an up-to-date understanding of PARP in DDR, the use of PARP inhibitors as mono- or combination therapies for cancer, and the potential of radiolabeled PARPi in clinical applications.

\section{PARP Regulation and Activation in Response to DNA Damage}

PARP enzymes form a 17-member group of mostly nuclear enzymes that share a conserved catalytic domain homologous to ADP-ribosyl-transferases diphtheria toxin (ARTD), which gives the PARP enzyme family its alternative name of ARTD-like enzymes (5). Upon DNA damage, members of the PARP family bind the DNA lesion, become catalytically active, and mediate ADP-ribosylation using $\mathrm{NAD}^{+}$as substrate, leading to rapid recruitment of other DNA repair factors (6). PARP-1, PARP-2 and tankyrase (PARP-5A/5B) produce poly-ADP-ribose (PAR) modifications on target proteins, while other PARPs are responsible for generating mono-ADP-ribose (MAR) and have potential cancer-related functions (7). The evidence to date suggests that only PARP-1, PARP-2 and PARP-3 are activated by DNA breaks and catalyze ADP-ribosylation after DNA damage, with PARP-1 responsible for $80-90 \%$ of global PAR synthesis in response to DNA damaging agents (6). Apart from SSB recognition, recent studies showed that PARP-1 can also detect 
DSB rapidly, and plays an essential role in regulation of DNA end resection for DSB repair, with recruitment and activation of PARP-1 occurring within $0.1 \mu$ s after induction of DSBs (6).

PARP-1 is the most studied of the PARP enzyme family by far. Its structure comprizes of three zinc finger domains for DNA binding, a BRCA-C-terminus domain (BRCT), a WGR (Trp-Gly-Arg) domain that contributes to but is not required for DNA interaction and catalytic activity regulation in response to DNA damage, and a catalytic domain consisting of a helical subdomain (HD) adjacent to a conserved ADP-ribosyl transferase fold (ART) (8). Compared to PARP-1 (N-terminal region: > 500 residues), PARP-2 and PARP-3 have more compact structures, with shorter Nterminal extensions (70 and 40 residues, respectively) from the WGR domain (Fig. 1A) (9). PARP-1 requires the zinc finger domains for DNA break detection and binding (10), but PARP-2 and PARP-3 rely on their WGR domain for this function $(2,11)$.

Despite differences in DNA damage detection, PARP-1, PARP-2 and PARP-3 share allosteric regulatory activation mechanisms, where binding to damaged DNA leads to an increase of PARylation activity (12). Langelier et al. demonstrated that the HD domain in PARP-1 can selectively and reversibly block or allow access of NAD ${ }^{+}$ or the NAD analogue BAD upon DNA binding (13). The HD domain, in its native state, was found primarily in a folded conformation, sterically blocking the catalytic binding site. Upon DNA damage detection, the binding of $\mathrm{NAD}^{+}$to the catalytic active site stabilizes the HD domain in its unfolded conformation and strengthens the interaction between the regulatory domains and DNA (Fig. 1B). This reversible blockage of the $\mathrm{NAD}^{+}$binding site provides control of $\mathrm{NAD}^{+}$access to the catalytic site, regulating the PARylation process. 


\section{PARP Trapping-Induced Cytotoxicity}

Clinical PARP inhibitors compete for binding in the NAD ${ }^{+}$binding pocket of the PARP enzymes, and are mostly selective for PARP-1 and PARP-2. However, different PARP inhibitors display markedly different levels of cytotoxicity, attributed to the ability of PARP inhibitors to prevent auto-PARylation of PARP, thereby preventing its dissociation from the broken DNA, thus stabilizing and trapping PARP on the DNA (14). This trapping leads to DSB formation by stalling and collapsing replication forks (15) or accelerating fork elongation (3). Hence, different degrees of trapping (talazoparib $>$ niraparib $\geq$ olaparib $=$ rucaparib $>$ veliparib $(16,17)$ ) lead to large differences in cellular toxicity. Although the trapping abilities of PARPi are dependent on catalytic inhibition and the resulting dissociation rates of PARPi $(17,18)$, reverse allostery effects also play a significant role. Molecular impacts of PARPi binding to PARP-1 corroborated by molecular dynamics simulation, hydrogen-exchange mass spectrometry (HXMS) and X-ray crystallography implied that different PARPi induce diverse degrees of structural deviations in the regulatory domains, thereby positively or negatively affecting the retention of PARP-1 trapped at the DNA damage site $(19,20)$. Three types of PARPi were discerned based on their allosteric impacts on the PARP-1-DNA complex (19): Type I, allosteric pro-retention on DNA, which induces HD conformational changes that reinforce contacts with the DNA break and result in slow release; Type II, with no or mild allosteric effects; and Type III, allosteric pro-release from DNA, which induce HD conformational changes opposite to type I inhibitors (Fig. 1C). These results have illuminated that the PARP-I trapping induced by some PARPi, such as type II PARPi (talazoparib and olaparib) and type III PARPi (rucaparib and niraparib), does not rely on the allosteric effect, 
indicating that other factors may contribute to the trapping ability of these PARPi, and therefore further investigation is warranted.

\section{PARP Inhibitors in Cancer Therapy - Preclinical and Clinical Development PARPi as Monotherapy}

The efficacy of PARPi monotherapy in HR deficient (HRD) cancers carrying $B R C A 1 / 2$ mutations was first demonstrated in cancer cell lines, and later confirmed in patient-derived xenograft (PDX) models. Treatment with olaparib resulted in significant tumor graft regression $(21,22)$ in PDX tumors of $B R C A 1$-mutant triple negative breast cancer (TNBC). Other alterations, such as absence of PTEN or ATM also cause sensitivity of TNBC (23) and small-cell lung cancer (SCLC) (24) to talazoparib treatment in vivo, confirming the effectiveness of PARPi beyond germline BRCA1/2 mutation cancers.

A wealth of clinical data supports the use of PARPi for maintenance treatment in platinum-sensitive high-grade serious ovarian cancer (HGSOC) patients. In the randomized, placebo-controlled, double blind phase 3 SOLO2 trial, patients with relapsed epithelial ovarian, fallopian tube or primary peritoneal cancer carrying a $B R C A 1 / 2$ mutation demonstrated a significantly longer median progression-free survival (PFS) under olaparib maintenance treatment than those receiving placebo (19.1 versus 5.5 months, 95\% CI 16.3-25.7 vs 5.2-5.8, HR 0.3), without significant detrimental effects on health-related quality of life (HRQoL) $(25,26)$.

The antitumor activity of olaparib was highlighted in the recent phase 3 SOLO3 (NCT02282020) and OlympiAD (27) trials, in germline BRCA-mutated ovarian cancer and HER2-negative breast cancer, respectively. Similarly, the ABRAZO study (28), and the latest EMBRACA trial, where patients underwent 
talazoparib monotherapy, showed a significant PFS advantage and higher objective response rate (ORR) over standard chemotherapy (8.6 versus 5.6 months, $62.6 \%$ versus $27.2 \%$, respectively) with greater HRQoL in favor of talazoparib (29). Several recently reported trials including POLO and PROfound also demonstrated the benefits of PARPi (olaparib or rucaparib) over placebo or androgen receptor (AR)-directed therapy, respectively, in metastatic pancreatic ductal adenocarcinoma (PDAC) patients harboring germline $B R C A$ mutation (30) and castration-resistant prostate cancer (mCRPC) patients with confirmed HRD phenotype (31). Ongoing efforts to expand the use of PARPi to a wider population of cancer patients in different settings beyond $B R C A$ mutation will undoubtedly expand the potential use of PARPi as monotherapy, and several studies are currently underway (NCT03402841, NCT03286842, NCT02000622).

\section{Combination Therapy with PARP Inhibitors}

Heterogeneous responses to single-agent PARPi are frequently observed in both preclinical models and clinical practice, even in $B R C A$-mutated tumors, associated with complex intrinsic or acquired resistance. The mechanisms of PARPi resistance are well-described in several recent reviews, with the majority related to restoration of HR capability (32). Rational combination treatment regimens thus become the next strategy to improve patient outcome e.g. using PARPi with chemotherapy, radiation, targeted drugs or immunotherapy. The choice of partner must be based on the fundamental insights into the molecular pathways that drive tumorigenesis and therapy response. Strategies to optimize the use of PARPi as chemopotentiators or radiosensitizers are being actively pursued to overcome or prevent the development of treatment resistance in patients. 
One approach is the combination of genotoxic agents and PARPi, where chemotherapy-induced DNA damage can increase the cellular reliance on DNA repair and hence sensitize tumors to PARPi, such as in (33). Combination of PARPi and ionizing radiation (IR) is another effective strategy to sensitize tumors to radiotherapy, without overt signs of toxicity at least in mice, warranting further its evaluation in ongoing clinical trials (34), despite an early recognition of the limitation of this combinatorial approach in cells (35). Given the relationship between PARP and HR repair (HRR) regulatory pathways, it is not surprising to see the potential benefits of combination treatments between PARPi and DDR signaling-targeting or otherwise genotoxic therapies. The increased reliance of cancer cells on alternative or specific DRR pathways provides an opportunity to pharmacologically induce HR deficiency, which in combination with PARPi achieves synergistic response. Disruption of for example, checkpoint kinase 1 (CHK1) activity with PARP inhibition leads to the accumulation of DSB and induces apoptosis following treatment of PDX mouse models using olaparib/MK8776 (36) or olaparib/prexasertib combinations. Tretinoin, used to treat acute promyelocytic leukemia patients, also acts as an effective HR disrupter and is capable of sensitizing $B R C A$-proficient TNBC to olaparib (38). Other studies involved the use of cyclic dependent kinase (CDK) inhibitors to disrupt DNA damage checkpoint signaling and prevent DNA repair in neuroendocrine prostate cancer (NEPC) (39) and TNBC PDX models (40). Inhibition of bromodomain-containing protein (BRD4) disrupts DDR signaling by reducing DNA repair proteins levels, thereby sensitizing cancer cells to olaparib in preclinical models $(41,42)$. Treatment of mCRPC PDXs with an androgen receptor (AR) inhibitor enzalutamide induces downregulation of AR-regulated HRR gene expression and sensitizes to PARPi (43). An overview is presented in Table 1. 
Promising efficacy of PARPi with genotoxic agents was observed in preclinical models and successfully translated in various cancer types: The phase 3 VELIA study demonstrated the efficacy of concurrent administration of veliparib and carboplatin with paclitaxel $(\mathrm{V} / \mathrm{CP})$ followed by veliparib maintenance in advanced HGSOC patients with and without germline $B R C A$ mutation. A significant PFS advantage was observed in both $B R C A$-mutant (34.7 versus 22 months, HR 0.44), HR defects cohorts (31.9 versus 20.5 months), and the intention-to-treat population (23.5 versus 17.3 months, HR 0.68) receiving veliparib versus placebo with chemotherapy (44). Similar efficacy was observed in breast cancer patients in phase 2 and 3 studies $(45,46)$.

Other clinical studies showed improved patient outcomes for treatment regimens involving PARPi combined with targeted drugs, such as anti-angiogenic and AR antagonists. The recent FDA approval of olaparib/bevacizumab combination therapy as a first-line maintenance treatment for HGSOC patients regardless of their HRD phenotypes based on the PAOLA-1 trial (47) will undoubtedly encourage additional studies to investigate other combination pairs (such as ICON9; NCT03278717, NCT02502266). For example, the use of olaparib/cediranib showed appreciable benefits in ovarian cancer patients (48). Other promising studies include the combination of abiraterone and olaparib in MCRPC patients, irrespective of HRR status (49). Combination of immune checkpoint inhibitors with PARPi is under active investigation, with only a limited amount of clinical data available to date. The MEDIOLA trial showed initial promise in a subset of ovarian cancer patients (platinum sensitive, germline $B R C A$-mutated) receiving this combination (50), but no improvement in ORR or PFS when a more heterogeneous patient population (without $B R C A$ mutation) was considered (51). Notwithstanding, the preliminary outcomes 
supported the tolerability of this combination in patients and better patient selections in subsequent trials should help to create clinically meaningful responses (NCT02484404). An overview is presented in Table 2.

\section{Radiolabeled PARP Inhibitors: Imaging and Therapy}

Molecular imaging using PARP imaging agents could serve as a powerful tool for functional imaging of PARP in vivo. Tagging a PARPi with a radionuclide allows PET $\left({ }^{11} \mathrm{C},{ }^{18} \mathrm{~F}\right)$ or SPECT $\left({ }^{123} \mathrm{I}\right)$ imaging to potential clinical benefits: interrogation of PARP expression levels, PARPi distribution, tumor uptake, and drug-target engagement in a non-invasive manner, which may allow tumor diagnosis and staging, selection of patient subgroups suitable for PARP inhibition, monitor the treatment response, or gauge the emergence of resistance. If, on the other hand, the same chemical scaffold is radiolabeled with a therapeutic radionuclide, emitting alpha $\left({ }^{211} \mathrm{At}\right)$, beta $\left({ }^{131} \mathrm{I}\right)$, or Auger $\left({ }^{123} \mathrm{I}\right)$ radiation, the resulting radionuclide therapy agent is capable of causing cell death by delivering ionizing radiation to PARP-expressing tumor cells

Over the past 15 years, numerous radiolabeled PARP agents have been developed (Fig. 2A) and undergone characterization in vitro and in animal models, with two compounds already evaluated in early phase clinical trials $(34,52) .\left[{ }^{11} \mathrm{C}\right] \mathrm{PJ} 34$ was the first radiolabeled PARPi, intended for PET imaging of tissue necrosis with upregulation of PARP-1 expression levels in a rat models of diabetes (53). The radiolabeled imaging agents $\left[{ }^{18} \mathrm{~F}\right] \mathrm{BO}(54),\left[{ }^{18} \mathrm{~F}\right] \mathrm{PARPi}(55),\left[{ }^{123 / 131} \mathrm{I}\right] \mathrm{PARPi}(56,57)$, $\left[{ }^{18} \mathrm{~F}\right]$ PARPi-FL (58) and $\left[{ }^{64} \mathrm{Cu}\right]$ PARPi-DOTA (59), all shared structural similarity to olaparib, and most displayed high specificity toward PARP. Also of note is the development of $\left[{ }^{18} \mathrm{~F}\right]$ olaparib, an ${ }^{18} \mathrm{~F}$-labeled isotopologue of olaparib, with an 
identical chemical structure as the parent molecule, hence with identical pharmacokinetic and dynamic profiles in vivo (60). Among the olaparib-derived radiotracers, $\left[{ }^{18} \mathrm{~F}\right] \mathrm{PARPi}-\mathrm{FL}$, the dual-modality PARP imaging agent (optical/PET), was used to image drug distribution and drug-target engagement of different PARP inhibitors in different cancer models $(61,62)$, demonstrating the potential application of radiolabeled-PARPi in monitoring of drug distribution and drug-target engagement. Rucaparib-inspired radiolabeled compounds $\left[{ }^{18} \mathrm{~F}\right] \mathrm{FE}-\mathrm{LS}-75$ (without publicly available preclinical characterization) (63), $\left[{ }^{125} \mathrm{I}\right] \mathrm{KX} 1$ (64), $\left[{ }^{125} \mathrm{I}\right] \mathrm{KX}-02-019$ (65), and $\left[{ }^{18} \mathrm{~F}\right] \mathrm{FTT}$ (66) also show excellent PARP selectivity.

Moving beyond preclinical models, both $\left[{ }^{18} \mathrm{~F}\right] \mathrm{FTT}(66,67)$ and $\left[{ }^{18} \mathrm{~F}\right] \mathrm{PARPi}$ $(55,68)$ have been evaluated in different types of cancers in clinical practice. $\left[{ }^{18} \mathrm{~F}\right] \mathrm{FTT}$ is currently the subject of phase 1 trials in ovarian patients (66) (Fig 2B), and also able to measure PARP-1 expression in different subtypes of breast cancer patients (estrogen receptor positive, human epidermal growth factor receptor 2-positive and triple negative) (67). $\left[{ }^{18} \mathrm{~F}\right] \mathrm{PARPi}$ is currently also in phase 1 trials but for head-andneck cancer (55) (Fig. 2C). Furthermore, recent clinical data has shown that the utility of $\left[{ }^{18} \mathrm{~F}\right]$ PARPi can be potentially expanded to image brain cancer in patients for treatment monitoring (68). In addition to applying radiolabeled-PARPi in patients for cancer detection, a fluorescent-labeled PARPi, PARPi-FL, was also subjected to clinical studies for the detection of oral carcinoma, and potentially serve as the surgical-margin assessment of epithelial cancer of the upper intestinal tract (69). These translational studies established the encouraging potential of PARP imaging agents in cancer detection for clinical practice.

Although these agents showed promising tumor uptake, they display different affinity and selectivity for PARP-1 and other isoforms, with a range of $\mathrm{IC}_{50}$ values 
$(2.3-200 \mathrm{nM})$ as a proxy for PARP affinity (Fig. 2A). Their selectivity toward other PARP family enzymes is not always fully characterized. Combined results indicate that small structural modifications may have significant impact on the target specificity of the compound. The Reiner group showed, for example, that altering the iodination position on the aromatic ring and the length of the linker between the iodinated-aromatic ring and the olaparib core resulted in very different $\mathrm{IC}_{50}$ values for PARP-1 (57). Similarly, recently reported on the effects of structure modification on tumor uptake and target specificity of the olaparib-derived radiolabeled PARPi $\left[{ }^{18} \mathrm{~F}\right]$ olaparib and $\left[{ }^{18} \mathrm{~F}\right] \mathrm{AZD} 2461(70)$.

The great potential of radiolabeled PARPi has now also expanded to the use of therapeutic radionuclides: ${ }^{123}$ I (Auger electron therapy) (71), ${ }^{131}$ I (high energy beta particles) (72), and ${ }^{211}$ At (alpha radiation) (73). These PARPi-based radionuclide therapy agents have shown some efficacy in brain cancer and neuroblastoma models with little toxicity to normal tissues, making use of the high ionizing potential and short ranges of these particle emitters for cancer treatment (Fig. 3A-3C).

To date, PARPi-derived radiolabeled compounds are based on either olapariblike structures or rucaparib-like structures. Given that small variations in structure can lead to large differences in PARP $\mathrm{IC}_{50}$ values and trapping, it may not be assumed that all radiolabeled variants are similar in PARP trapping ability, or elicit the same allosteric effects under the same circumstances. Olaparib and rucaparib have a comparable ability for trapping PARP-1 on DNA via catalytic inhibition, yet their allosteric effects differ significantly, and may even be different for other PARP isoforms. These variances may result in diverse degrees of target engagement and PARP trapping, including PARP inhibitor trapping. This may in turn influence tumor versus normal tissue uptake, affecting imaging contrast and toxicity, of radionuclide 
imaging and therapy. Therefore, target specificity and PARP trapping ability of PARPi or their radiolabeled analogues, and target expression should be taken into account in PARP-targeting approaches. For example, high accumulation of ${ }^{18} \mathrm{~F}$ PARPi in healthy tissues such as lymph nodes, bone marrow, liver and spleen may induce higher toxicity to these organs due to high physiological expression of PARP1 and PARP-2 (55). Careful consideration is needed in the interpretation of PARPiderived imaging agents, which cannot necessarily predict the precise behavior of analogous, but non-identical radionuclide therapy compounds. This can of course be overcome by using thera(g)nostic compounds, which allow imaging patient dosimetry calculation and therapy response, as demonstrated by the recent study using $\left[{ }^{123} \mathrm{I}\right]$ PARPi in glioblastoma models (Fig. 3A) (71).

\section{Conclusions and Future Prospects}

The discovery and development of PARPi in BRCA-mutant cancers is arguably the most elegant example of synthetic lethality to target tumor suppression gene loss in clinical practice. PARP inhibitor therapy can be of considerable benefit to a wide variety of cancer patients as a monotherapy and can synergize with appropriately selected drugs to improve treatment outcome. However, before the full potential of mono- and combination therapies may be achieved in clinical practice, it is imperative to recognize the limitations currently faced in the PARP inhibition in cancer therapy. Attention should be paid to identification and validation of predictive biomarkers to predict synthetic lethality, how distinct molecular mechanisms of action of different PARPi, off-target effects due to high physiological PARP-expressing healthy organs, tumor heterogeneity and genetic modifications in patients may lead to unexpected treatment responses. Genetic testing e.g. BRACAnalysis ${ }^{\circledR}$ or myChoice ${ }^{\circledR}$ 
test (Myriad Genetics) to select those patients who will benefit from PARPi treatment has been implemented in many clinical trials, and the use of diagnostic PARPi-based PET imaging may serve as a non-invasive method to monitor patient treatment response or study the degree of drug engagement in tumor (based on PARP-1 expression levels) at any time during the treatment cycle. Proof-of-concept studies using imaging agents to study PARP levels will add value to the interpretation of clinical data. The emergence of PARP-targeting radionuclide therapy represents another treatment strategy that warrants further investigation to explore its potential.

\section{Authors' Contribution}

C.Y.C., K.V.T. and B.C. contributed substantially to the design, collecting literature data and writing the article. All authors discussed, reviewed and approved the final manuscript before submission. 


\section{References}

1. Min A, Im S-A. PARP inhibitors as therapeutics: beyond modulation of PARylation. Cancers 2020; $12: 394$.

2. Pascal JM. The comings and goings of PARP-1 in response to DNA damage. DNA repair 2018; 71:177-82.

3. Maya-Mendoza A, Moudry P, Merchut-Maya JM, Lee M, Strauss R, Bartek J. High speed of fork progression induces DNA replication stress and genomic instability. Nature 2018;559:279-84.

4. Cortés - Ledesma F, Aguilera A. Double - strand breaks arising by replication through a nick are repaired by cohesin - dependent sister - chromatid exchange. EMBO reports 2006; 7:919-26.

5. Palazzo L, Mikoč A, Ahel I. ADP - ribosylation: new facets of an ancient modification. FEBS J 2017;284:2932-46.

6. Caron M-C, Sharma AK, O'Sullivan J, Myler LR, Ferreira MT, Rodrigue A, et al. Poly (ADP-ribose) polymerase-1 antagonizes DNA resection at doublestrand breaks. Nat Commun 2019;10:1-16.

7. Vyas S, Chang P. New PARP targets for cancer therapy. Nat Rev Cancer 2014; $14: 502-9$.

8. Langelier M-F, Planck JL, Roy S, Pascal JM. Structural basis for DNA damage-dependent poly (ADP-ribosyl) ation by human PARP-1. Science 2012;336:728-32.

9. Riccio AA, Cingolani G, Pascal JM. PARP-2 domain requirements for DNA damage-dependent activation and localization to sites of DNA damage. Nucleic Acids Res 2016;44:1691-702.

10. Gagné J-P, Rouleau M, Poirier GG. PARP-1 Activation-Bringing the Pieces Together. Science 2012;336:678-9.

11. Obaji E, Haikarainen T, Lehtiö L. Structural basis for DNA break recognition by ARTD2/PARP2. Nucleic Acids Res 2018;46:12154-65.

12. Langelier M-F, Riccio AA, Pascal JM. PARP-2 and PARP-3 are selectively activated by $5^{\prime}$ phosphorylated DNA breaks through an allosteric regulatory mechanism shared with PARP-1. Nucleic Acids Res 2014;42:7762-75.

13. Langelier M-F, Zandarashvili L, Aguiar PM, Black BE, Pascal JM. NAD+ analog reveals PARP-1 substrate-blocking mechanism and allosteric communication from catalytic center to DNA-binding domains. Nat Commun 2018;9:1-13.

14. Murai J, Pommier Y. PARP trapping beyond homologous recombination and platinum sensitivity in cancers. Annu Rev Cancer Biol 2019;3:131-50. 
15. Prasad CB, Prasad SB, Yadav SS, Pandey LK, Singh S, Pradhan S, et al. Olaparib modulates DNA repair efficiency, sensitizes cervical cancer cells to cisplatin and exhibits anti-metastatic property. Sci Rep 2017;7:1-15.

16. Murai J, Shar-yin NH, Das BB, Renaud A, Zhang Y, Doroshow JH, et al. Trapping of PARP1 and PARP2 by clinical PARP inhibitors. Cancer Res 2012;72:5588-99.

17. Murai J, Huang S-YN, Renaud A, Zhang Y, Ji J, Takeda S, et al. Stereospecific PARP trapping by BMN 673 and comparison with olaparib and rucaparib. Mol Cancer Ther 2014;13:433-43.

18. Hopkins TA, Shi Y, Rodriguez LE, Solomon LR, Donawho CK, DiGiammarino EL, et al. Mechanistic dissection of PARP1 trapping and the impact on in vivo tolerability and efficacy of PARP inhibitors. Mol Cancer Res 2015; 13:1465-77.

19. Zandarashvili L, Langelier M-F, Velagapudi UK, Hancock MA, Steffen JD, Billur $\mathrm{R}$, et al. Structural basis for allosteric PARP-1 retention on DNA breaks. Science 2020;368: eaax6367.

20. Kumar C, PTV L, Arunachalam A. A mechanistic approach to understand the allosteric reverse signaling by selective and trapping poly (ADP-ribose) polymerase 1 (PARP-1) inhibitors. J Biomol Struct Dyn 2020;38:2482-92.

21. Ter Brugge P, Kristel P, Van Der Burg E, Boon U, De Maaker M, Lips E, et al. Mechanisms of therapy resistance in patient-derived xenograft models of BRCA1-deficient breast cancer. J Natl Cancer I 2016;108:djw148.

22. Park HS, Lee JD, Kim JY, Park S, Kim JH, Han HJ, et al. Establishment of chemosensitivity tests in triple-negative and BRCA-mutated breast cancer patient-derived xenograft models. Plos one 2019; 14:e0225082.

23. Evans KW, Yuca E, Akcakanat A, Scott SM, Arango NP, Zheng X, et al. A population of heterogeneous breast cancer patient-derived xenografts demonstrate broad activity of PARP inhibitor in BRCA1/2 wild-type tumors. Clin Cancer Res 2017;23:6468-77.

24. Stewart CA, Tong P, Cardnell RJ, Sen T, Li L, Gay CM, et al. Dynamic variations in epithelial-to-mesenchymal transition (EMT), ATM, and SLFN11 govern response to PARP inhibitors and cisplatin in small cell lung cancer. Oncotarget 2017;8:28575-87.

25. Pujade-Lauraine E, Ledermann JA, Selle F, Gebski V, Penson RT, Oza AM, et al. Olaparib tablets as maintenance therapy in patients with platinumsensitive, relapsed ovarian cancer and a BRCA1/2 mutation (SOLO2/ENGOTOv21): a double-blind, randomised, placebo-controlled, phase 3 trial. Lancet Oncol 2017; 18:1274-84.

26. Friedlander M, Gebski V, Gibbs E, Davies L, Bloomfield R, Hilpert F, et al. Health-related quality of life and patient-centred outcomes with olaparib maintenance after chemotherapy in patients with platinum-sensitive, relapsed 
ovarian cancer and a BRCA1/2 mutation (SOLO2/ENGOT Ov-21): a placebocontrolled, phase 3 randomised trial. Lancet Oncol 2018;19:1126-34.

27. Robson M, Im S-A, Senkus E, Xu B, Domchek SM, Masuda N, et al. Olaparib for metastatic breast cancer in patients with a germline BRCA mutation. $\mathrm{N}$ Engl J Med 2017;377:523-33.

28. Turner NC, Telli ML, Rugo HS, Mailliez A, Ettl J, Grischke E-M, et al. A phase II study of talazoparib after platinum or cytotoxic nonplatinum regimens in patients with advanced breast cancer and germline BRCA1/2 mutations (ABRAZO). Clin Cancer Res 2019;25:2717-24.

29. Litton JK, Rugo HS, Ettl J, Hurvitz SA, Gonçalves A, Lee K-H, et al. Talazoparib in patients with advanced breast cancer and a germline BRCA mutation. N Engl J Med 2018;379:753-63.

30. Golan T, Hammel P, Reni M, Van Cutsem E, Macarulla T, Hall MJ, et al. Maintenance olaparib for germline BRCA-mutated metastatic pancreatic cancer. N Engl J Med 2019;381:317-27.

31. de Bono J, Mateo J, Fizazi K, Saad F, Shore N, Sandhu S, et al. Olaparib for metastatic castration-resistant prostate cancer. N Engl J Med 2020;382:2091102.

32. Noordermeer SM, van Attikum H. PARP inhibitor resistance: a tug-of-war in BRCA-mutated cells. Trends Cell Biol 2019;29:820-34.

33. Lok BH, Gardner EE, Schneeberger VE, Ni A, Desmeules P, Rekhtman N, et al. PARP inhibitor activity correlates with SLFN11 expression and demonstrates synergy with temozolomide in small cell lung cancer. Clin Cancer Res 2017;23:523-35.

34. Jannetti SA, Zeglis BM, Zalutsky MR, Reiner T. Poly (ADP-Ribose) Polymerase (PARP) Inhibitors and Radiation Therapy. Front Pharmacol 2020;11:170.

35. Chalmers A, Johnston P, Woodcock M, Joiner M, Marples B. PARP-1, PARP-2, and the cellular response to low doses of ionizing radiation. Int $\mathrm{J}$ Radiat Oncol Biol Phys 2004;58:410-9.

36. Kim H, George E, Ragland RL, Rafail S, Zhang R, Krepler C, et al. Targeting the ATR/CHK1 axis with PARP inhibition results in tumor regression in BRCA-mutant ovarian cancer models. Clin Cancer Res 2017;23:3097-108.

37. Parmar K, Kochupurakkal BS, Lazaro J-B, Wang ZC, Palakurthi S, Kirschmeier PT, et al. The CHK1 inhibitor prexasertib exhibits monotherapy activity in high-grade serous ovarian cancer models and sensitizes to PARP inhibition. Clin Cancer Res 2019;25:6127-40.

38. Luo M-L, Zheng F, Chen W, Liang Z-M, Chandramouly G, Tan J, et al. Inactivation of the prolyl isomerase Pin1 sensitizes BRCA1-proficient breast cancer to PARP inhibition. Cancer Res 2020;80:3033-45. 
39. Liu B, Li L, Yang G, Geng $\mathrm{C}$, Luo $\mathrm{Y}, \mathrm{Wu} \mathrm{W}$, et al. PARP Inhibition Suppresses GR-MYCN-CDK5-RB1-E2F1 Signaling and Neuroendocrine Differentiation in Castration-Resistant Prostate Cancer. Clin Cancer Res 2019;25:6839-51.

40. Johnson SF, Cruz C, Greifenberg AK, Dust S, Stover DG, Chi D, et al. CDK12 inhibition reverses de novo and acquired PARP inhibitor resistance in BRCA wild-type and mutated models of triple-negative breast cancer. Cell Rep 2016; 17:2367-81.

41. Sun C, Yin J, Fang Y, Chen J, Jeong KJ, Chen X, et al. BRD4 inhibition is synthetic lethal with PARP inhibitors through the induction of homologous recombination deficiency. Cancer cell 2018;33:401-16.

42. Miller AL, Fehling SC, Garcia PL, Gamblin TL, Council LN, van Waardenburg RC, et al. The BET inhibitor JQ1 attenuates double-strand break repair and sensitizes models of pancreatic ductal adenocarcinoma to PARP inhibitors. EBioMedicine 2019;44:419-30.

43. Li L, Karanika S, Yang G, Wang J, Park S, Broom B, et al. Enzalutamideinduced "BRCAness" and PARP inhibition is a synthetic lethal therapy for castration-resistant prostate cancer. Sci Signal 2017;10:1-23.

44. Coleman RL, Fleming GF, Brady MF, Swisher EM, Steffensen KD, Friedlander $\mathrm{M}$, et al. Veliparib with first-line chemotherapy and as maintenance therapy in ovarian cancer. N Engl J Med 2019;381:2403-15.

45. Loibl S, O'Shaughnessy J, Untch M, Sikov WM, Rugo HS, McKee MD, et al. Addition of the PARP inhibitor veliparib plus carboplatin or carboplatin alone to standard neoadjuvant chemotherapy in triple-negative breast cancer (BrighTNess): a randomised, phase 3 trial. Lancet Oncol 2018;19:497-509.

46. Han H, Diéras V, Robson M, Palácová M, Marcom P, Jager A, et al. Veliparib with temozolomide or carboplatin/paclitaxel versus placebo with carboplatin/paclitaxel in patients with BRCA1/2 locally recurrent/metastatic breast cancer: randomized phase II study. Ann Oncol 2018;29:154-61.

47. Ray-Coquard I, Pautier P, Pignata S, Pérol D, González-Martín A, Berger R, et al. Olaparib plus bevacizumab as first-line maintenance in ovarian cancer. N Engl J Med 2019;381:2416-28.

48. Liu J, Barry W, Birrer M, Lee J-M, Buckanovich R, Fleming G, et al. Overall survival and updated progression-free survival outcomes in a randomized phase II study of combination cediranib and olaparib versus olaparib in relapsed platinum-sensitive ovarian cancer. Ann Oncol 2019;30:551-7.

49. Clarke N, Wiechno P, Alekseev B, Sala N, Jones R, Kocak I, et al. Olaparib combined with abiraterone in patients with metastatic castration-resistant prostate cancer: a randomised, double-blind, placebo-controlled, phase 2 trial. Lancet Oncol 2018; 19:975-86. 
50. Drew Y, De Jonge M, Hong S, Park Y, Wolfer A, Brown J, et al. An openlabel, phase II basket study of olaparib and durvalumab (MEDIOLA): results in germline BRCA-mutated (gBRCAm) platinum-sensitive relapsed (PSR) ovarian cancer (OC). Gynecol Oncol 2018;149:246-7.

51. Lampert EJ, Zimmer AS, Padget MR, Cimino-Mathews A, Nair JR, Liu Y, et al. Combination of PARP inhibitor olaparib, and PD-L1 inhibitor durvalumab, in recurrent ovarian cancer: a proof-of-concept phase 2 study. Clin Cancer Res 2020;26:4268-79.

52. Carney B, Kossatz S, Reiner T. Molecular imaging of PARP. J Nucl Med 2017;58:1025-30.

53. Tu Z, Chu W, Zhang J, Dence CS, Welch MJ, Mach RH. Synthesis and in vivo evaluation of [11C] PJ34, a potential radiotracer for imaging the role of PARP-1 in necrosis. Nucl Med Biol 2005;32:437-43.

54. Reiner T, Lacy J, Keliher EJ, Yang KS, Ullal A, Kohler RH, et al. Imaging therapeutic PARP inhibition in vivo through bioorthogonally developed companion imaging agents. Neoplasia 2012;14:169-77.

55. Schöder HM, França PDDS, Nakajima R, Burnazi EM, Roberts S, Brand C, et al. Safety and feasibility of PARP1/2 imaging with 18F-PARPi in patients with head and neck cancer. Clin Cancer Res 2020;26:3110-6.

56. Zmuda F, Malviya G, Blair A, Boyd M, Chalmers AJ, Sutherland A, et al. Synthesis and evaluation of a radioiodinated tracer with specificity for poly (ADP-ribose) polymerase-1 (PARP-1) in vivo. J Med Chem 2015;58:8683-93.

57. Salinas B, Irwin CP, Kossatz S, Bolaender A, Chiosis G, Pillarsetty N, et al. Radioiodinated PARP1 tracers for glioblastoma imaging. EJNMMI Res 2015;5:1-14.

58. Carlucci G, Carney B, Brand C, Kossatz S, Irwin CP, Carlin SD, et al. Dualmodality optical/PET imaging of PARP1 in glioblastoma. Mol Imaging Biol 2015; $17: 848-55$.

59. Huang T, Hu P, Banizs AB, He J. Initial evaluation of Cu-64 labeled PARPiDOTA PET imaging in mice with mesothelioma. Bioorg Med Chem Lett 2017;27:3472-6.

60. Wilson TC, Xavier M-A, Knight J, Verhoog S, Torres JB, Mosley M, et al. PET Imaging of PARP Expression Using 18F-Olaparib. J Nucl Med 2019;60:504-10.

61. Carney B, Kossatz S, Lok BH, Schneeberger V, Gangangari KK, Pillarsetty NVK, et al. Target engagement imaging of PARP inhibitors in small-cell lung cancer. Nat Commun 2018;9:1-13.

62. Kossatz S, Carney B, Farley C, Weber WA, Drain CM, Reiner T. Direct imaging of drug distribution and target engagement of the PARP inhibitor rucaparib. J Nucl Med 2018;59:1316-20. 
63. Riss PJ, Soskic V, Schrattenholz A, Roesch F. Synthesis and radiosynthesis of N5 - $[18 \mathrm{~F}]$ fluoroethyl - Pirenzepine and its metabolite N5 - [18F] fluoroethyl - LS 75. J Labelled Compd Radiopharm 2009;52:576-9.

64. Makvandi M, Xu K, Lieberman BP, Anderson R-C, Effron SS, Winters HD, et al. A radiotracer strategy to quantify PARP-1 expression in vivo provides a biomarker that can enable patient selection for PARP inhibitor therapy. Cancer Res 2016;76:4516-24.

65. Anderson R-C, Makvandi M, Xu K, Lieberman BP, Zeng C, Pryma DA, et al. Iodinated benzimidazole PARP radiotracer for evaluating PARP1/2 expression in vitro and in vivo. Nucl Med Biol 2016;43:752-8.

66. Makvandi M, Pantel A, Schwartz L, Schubert E, Xu K, Hsieh C-J, et al. A PET imaging agent for evaluating PARP-1 expression in ovarian cancer. $\mathrm{J}$ Clin Invest 2018; 128:2116-26.

67. McDonald ES, Doot RK, Pantel AR, Farwell MD, Mach RH, Maxwell KN, et al. Positron Emission Tomography Imaging of Poly-(Adenosine Diphosphate-Ribose) Polymerase 1 Expression in Breast Cancer: A Nonrandomized Clinical Trial. JAMA oncology 2020;6:921-3.

68. Young RJ, de Souza Franca PD, Pirovano G, Piotrowski AF, Nicklin PJ, Riedl $\mathrm{CC}$, et al. Preclinical and first-in-human-brain-cancer applications of [18F] poly-(ADP-ribose) polymerase inhibitor PET/MR. medRxiv 2020:doi: https://doi.org/10.1101/2020.07.13.20141036.

69. Kossatz S, Pirovano G, França PDDS, Strome AL, Sunny SP, Zanoni DK, et al. Validation of the use of a fluorescent PARP1 inhibitor for the detection of oral, oropharyngeal and oesophageal epithelial cancers. Nat Biomed Eng 2020; $4: 272-85$.

70. Guibbal F, Hopkins SL, Pacelli A, Isenegger PG, Mosley M, Torres JB, et al. [18F] AZD2461, an Insight on Difference in PARP Binding Profiles for DNA Damage Response PET Imaging. Mol Imaging Biol 2020:DOI: 10.1007/s11307-020-01497-6.

71. Pirovano G, Jannetti SA, Carter LM, Sadique A, Kossatz S, Guru N, et al. Targeted brain tumor radiotherapy using an Auger emitter. Clin Cancer Res 2020;26:2871-81.

72. Jannetti SA, Carlucci G, Carney B, Kossatz S, Shenker L, Carter LM, et al. PARP-1-Targeted Radiotherapy in Mouse Models of Glioblastoma. J Nucl Med 2018;59:1225-33.

73. Makvandi M, Lee H, Puentes LN, Reilly SW, Rathi KS, Weng C-C, et al. Targeting PARP-1 with Alpha-Particles Is Potently Cytotoxic to Human Neuroblastoma in Preclinical Models. Mol Cancer Ther 2019;18:1195-204. 
74. De Koning L, Decaudin D, El Botty R, Nicolas A, Carita G, Schuller M, et al. PARP inhibition increases the response to chemotherapy in uveal melanoma. Cancers 2019;11:1-16.

75. Heisey DA, Lochmann TL, Floros KV, Coon CM, Powell KM, Jacob S, et al. The ewing family of tumors relies on BCL-2 and BCL-XL to escape PARP inhibitor toxicity. Clin Cancer Res 2019;25:1664-75.

76. Santiago - O’ Farrill JM, Weroha SJ, Hou X, Oberg AL, Heinzen EP, Maurer MJ, et al. Poly (adenosine diphosphate ribose) polymerase inhibitors induce autophagy - mediated drug resistance in ovarian cancer cells, xenografts, and patient - derived xenograft models. Cancer 2020;126:894-907.

77. Ordóñez JL, Amaral AT, Carcaboso AM, Herrero-Martín D, del Carmen García-Macías M, Sevillano V, et al. The PARP inhibitor olaparib enhances the sensitivity of Ewing sarcoma to trabectedin. Oncotarget 2015;6:18875-90.

78. Cruz C, Castroviejo-Bermejo M, Gutiérrez-Enríquez S, Llop-Guevara A, Ibrahim Y, Gris-Oliver A, et al. RAD51 foci as a functional biomarker of homologous recombination repair and PARP inhibitor resistance in germline BRCA-mutated breast cancer. Ann Oncol 2018;29:1203-10.

79. AlHilli MM, Becker MA, Weroha SJ, Flatten KS, Hurley RM, Harrell MI, et al. In vivo anti-tumor activity of the PARP inhibitor niraparib in homologous recombination deficient and proficient ovarian carcinoma. Gynecol Oncol 2016;143:379-88.

80. Lohse I, Kumareswaran R, Cao P, Pitcher B, Gallinger S, Bristow RG, et al. Effects of combined treatment with ionizing radiation and the PARP inhibitor olaparib in BRCA mutant and wild type patient-derived pancreatic cancer xenografts. PloS one 2016; 11:e0167272.

81. Yun E-J, Lin C-J, Dang A, Hernandez E, Guo J, Chen W-M, et al. Downregulation of Human DAB2IP Gene Expression in Renal Cell Carcinoma Results in Resistance to Ionizing Radiation. Clin Cancer Res 2019; $25: 4542-51$.

82. Laird JH, Lok BH, Ma J, Bell A, de Stanchina E, Poirier JT, et al. Talazoparib is a potent radiosensitizer in small cell lung cancer cell lines and xenografts. Clin Cancer Res 2018;24:5143-52.

83. Jue TR, Nozue K, Lester AJ, Joshi S, Schroder LB, Whittaker SP, et al. Veliparib in combination with radiotherapy for the treatment of MGMT unmethylated glioblastoma. J Transl Med 2017;15:1-8.

84. Yin Y, Shen Q, Zhang $\mathrm{P}$, Tao R, Chang W, Li R, et al. Chk1 inhibition potentiates the therapeutic efficacy of PARP inhibitor BMN673 in gastric cancer. Am J Cancer Res 2017;7:473-83. 
85. Parvin S, Ramirez-Labrada A, Aumann S, Lu X, Weich N, Santiago G, et al. LMO2 confers synthetic lethality to PARP inhibition in DLBCL. Cancer cell 2019;36:237-49.

86. Fok JH, Ramos-Montoya A, Vazquez-Chantada M, Wijnhoven PW, Follia V, James N, et al. AZD7648 is a potent and selective DNA-PK inhibitor that enhances radiation, chemotherapy and olaparib activity. Nat Commun 2019; $10: 1-15$.

87. Zhang W, Liu B, Wu W, Li L, Broom BM, Basourakos SP, et al. Targeting the MYCN-PARP-DNA damage response pathway in neuroendocrine prostate cancer. Clin Cancer Res 2018;24:696-707.

88. Oza AM, Cibula D, Benzaquen AO, Poole C, Mathijssen RH, Sonke GS, et al. Olaparib combined with chemotherapy for recurrent platinum-sensitive ovarian cancer: a randomised phase 2 trial. Lancet Oncol 2015;16:87-97.

89. Pietanza MC, Waqar SN, Krug LM, Dowlati A, Hann CL, Chiappori A, et al. Randomized, double-blind, phase II study of temozolomide in combination with either veliparib or placebo in patients with relapsed-sensitive or refractory small-cell lung cancer. J Clin Oncol 2018;36:2386-94.

90. Owonikoko TK, Dahlberg SE, Sica GL, Wagner LI, Wade III JL, Srkalovic G, et al. Randomized phase II trial of cisplatin and etoposide in combination with veliparib or placebo for extensive-stage small-cell lung cancer: ECOGACRIN 2511 study. J Clin Oncol 2019;37:222-9. 


\section{Figure Legends}

Figure 1: Allosteric regulation of PARP-1 and effects on PARP-1 allostery by PARPi.

(A) Schematic representation of multi-domain organization of human PARP-1, PARP-2, and PARP-3. Zinc fingers (Zn1, Zn2, and Zn3); BRCT (BRCA Cterminus); WGR (Trp-Gly-Arg domain); HD (helical domain); ART (ADPribosyltransferase).

(B) Overall mechanism and allosteric regulation of PARP-1 (13). Upon DNA damage, the multi-domains of PARP-1 quickly assemble on the DNA damaged site. The binding of $\mathrm{NAD}^{+}$to the catalytic domain promotes PARylation to produce PAR chains for the recruitment of target proteins (such as DNA ligase, XRCC1, etc.). Auto-PARylation leads to dissociation of PARP-1 from the DNA to allow binding of other repair proteins to the DNA; The HD domain regulates $\mathrm{NAD}^{+}$access and catalytic activity of PARP-1 through a reversible substrate-blocking mechanism. In the absence of damaged DNA, the HD is in folded conformation and sterically blocks the substrate-binding site (the nicotinamide site $(\mathrm{N})$ and the adenosine site $(\mathrm{A})$ ) to restrict the access of $\mathrm{NAD}^{+}$. The assembly of DNA-binding domains of PARP-1 on the DNA break interfaces with the HD domain, which promotes an unfolded $\mathrm{HD}$ conformation to restore access of $\mathrm{NAD}^{+}$to the catalytic active site. The $\mathrm{NAD}^{+}$binding to the catalytic site drives the $\mathrm{HD}$ equilibrium distribution to its unfolded state (purple arrow), thus strengthening the interaction between the regulatory domains and the DNA break.

(C) Impacts on PARP-1 allostery in DDR by PARPi binding (19). Three distinct types of PARPi based on their allosteric effects on the PARP-1/SSB DNA 
complex: Type I PARPi influence PARP-1 allostery and retain PARP-1 on DNA (in green), whereas type III PARPi perturb PARP-1 allostery and release PARP-1 from DNA (in red). Type II PARPi do not influence PARP-1 allostery (in black).

Figure 2: Molecular structures and PARP-1 $\mathrm{IC}_{50}$ or inhibition constant $\left(\mathrm{K}_{\mathrm{i}}\right)$ values of PARP-targeted PET imaging agents, and examples of their clinical studies.

(A) Molecular structures with radionuclide highlighted in red, and PARP-1 IC 50 or inhibition constant $\left(\mathrm{K}_{\mathrm{i}}\right)$ values of PARP-targeted PET imaging agents.

(B) Clinical $\left[{ }^{18} \mathrm{~F}\right] \mathrm{FTT}$ and $\left[{ }^{18} \mathrm{~F}\right] \mathrm{FDG}$ PET/CT images of a patient completed 4 cycles of carboplatin and paclitaxel (66). A patient with omental metastases showed higher uptake of $\left[{ }^{18} \mathrm{~F}\right] \mathrm{FTT}$ than $\left[{ }^{18} \mathrm{~F}\right] \mathrm{FDG}$ prior $\left(\mathrm{SUV}_{\max }: 5.1\right.$ vs 2.0$)$ and after ( $\mathrm{SUV}_{\max }: 7.8$ vs 3.4) chemotherapy. Yellow arrowheads indicate sites of disease. Reproduced with permission.

(C) A 67-year-old patient with oropharyngeal squamous cell carcinoma (OPSCC) underwent $\left[{ }^{18} \mathrm{~F}\right] \mathrm{PARPi}$ PET/CT imaging (55). Maximum intensity projections at $30 \mathrm{~min}, 60 \mathrm{~min}$ and $120 \mathrm{~min}$ post $\left[{ }^{18} \mathrm{~F}\right] \mathrm{PARPi}$ injection in the patient with OPSCC. At $120 \mathrm{~min}$, uptake in the primary tumor was higher than lymph nodes ( $\mathrm{SUV}_{\max }: 4.1$ vs 3.6). Green arrow: primary tumor; Red arrow: metastatic lymph node. Reproduced with permission.

Figure 3: PARP-targeted therapeutic agents and their preclinical evaluations.

(A) In vivo $\left[{ }^{123} \mathrm{I}\right] \mathrm{MAPi}$ SPECT/CT imaging and $\left[{ }^{123} \mathrm{I}\right] \mathrm{MAPi}$ efficacy (71). Molecular structure of $\left[{ }^{123} \mathrm{I}\right] \mathrm{MAPi}$; SPECT/CT images of orthotopic GBM model were taken at $18 \mathrm{~h}$ after injecting with $\left[{ }^{123} \mathrm{I}\right] \mathrm{MAPi}(\mathrm{T}=$ tumor, $\mathrm{Cl}=$ clearing organs), showing specific tumor uptake of $\left[{ }^{123} \mathrm{I}\right] \mathrm{MAPi}$; Kaplan-Meier 
survival curves of vehicle or $\left[{ }^{123} \mathrm{I}\right] \mathrm{MAPi}$ (single injection: $0.37-1.11 \mathrm{MBq}$ ) treated mice. $\mathrm{P}<0.01 .\left[{ }^{123} \mathrm{I}\right] \mathrm{MAPi}$ treated mice showed improved survival compared to the vehicle treated mice; Cytology staining and MRI imaging of brain tissues of untreated mouse at week 3 , vehicle treated mouse at week 7 and $\left[{ }^{123} \mathrm{I}\right] \mathrm{MAPi}$ treated mouse at 14 week after tumor implantation. Reproduced with permission.

(B) In vivo therapeutic effect of $\left[{ }^{131} \mathrm{I}\right] \mathrm{PARPi}$ in subcutaneous GBM mouse models (72). Molecular structure of $\left[{ }^{131} \mathrm{I}\right] \mathrm{PARPi}$; Tumor growth at week 0 and week 2 of the mice treated with PBS, $\left[{ }^{127} \mathrm{I}\right] \mathrm{PARPi}$ or $\left[{ }^{131} \mathrm{I}\right] \mathrm{PARPi}$. The treatment with ${ }^{131}$ I]PARPi suppressed tumor growth $\mathrm{P}<0.01$. Reproduced with permission.

(C) In vivo comparison of single and fractional dosing regimens of $\left[{ }^{211} \mathrm{At}\right] \mathrm{MM} 4$ in IMR-05 xenograft models (73). Molecular structure of [ [ $\left.{ }^{211} \mathrm{At}\right] \mathrm{MM} 4$; Tumor growth and Kaplan-Meier curves for IMR-05 tumor-bearing mice treated with single high dose of $1,480 \mathrm{kBq}$ vs. a fractionated dose of $370 \mathrm{kBq}$ twice weekly for a cumulative dose of $1,480 \mathrm{kBq}$; Immunofluorescence staining for PARP-1 in tumor sections harvested from IMR-05 tumor-bearing mice treated with 370 $\mathrm{kBq}$ of $\left[{ }^{211} \mathrm{At}\right] \mathrm{MM} 4$ at 24,48 , and $72 \mathrm{~h}$ after treatment, showing upregulation of PARP-1 compared to the control. Reproduced with permission.

Table 1: Combination therapies with PARP inhibitors in PDX models.

Table 2: Selected clinical trials evaluating PARP inhibitors combinations. 
Figures: Figure 1

Catalytic domain

A
PARP-1
$\mathrm{N}-\mathrm{Zn} 1-\mathrm{Zn} 2-\mathrm{Zn} 3-\mathrm{BRCT}$
WGR - HD ART -C
PARP-2/PARP-3

$$
\mathrm{N}-\text { WGR }-\mathrm{HD} \text { ART }-\mathrm{C}
$$

B

HD in folded

conformation selectively

block $\mathrm{NAD}^{+}$binding to the

substrate-binding site of

ART domain

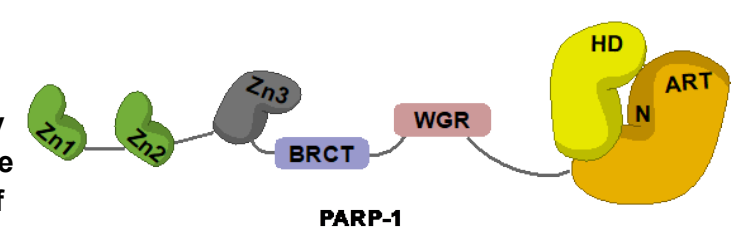

Binding to

damaged DNA

DNA-binding domains of PARP-1 assemble on DNA

break with dynamic

HD conformation
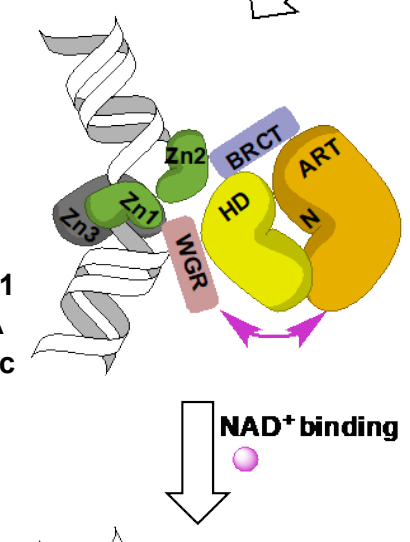

\section{NAD ${ }^{+}$binding stabilizes the} distorted HD (unfold conformation), reinforcing multidomains interaction with DNA break

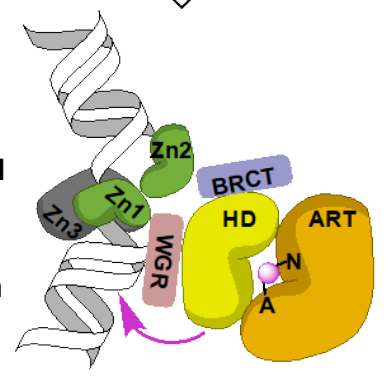

Reserve allostery
C
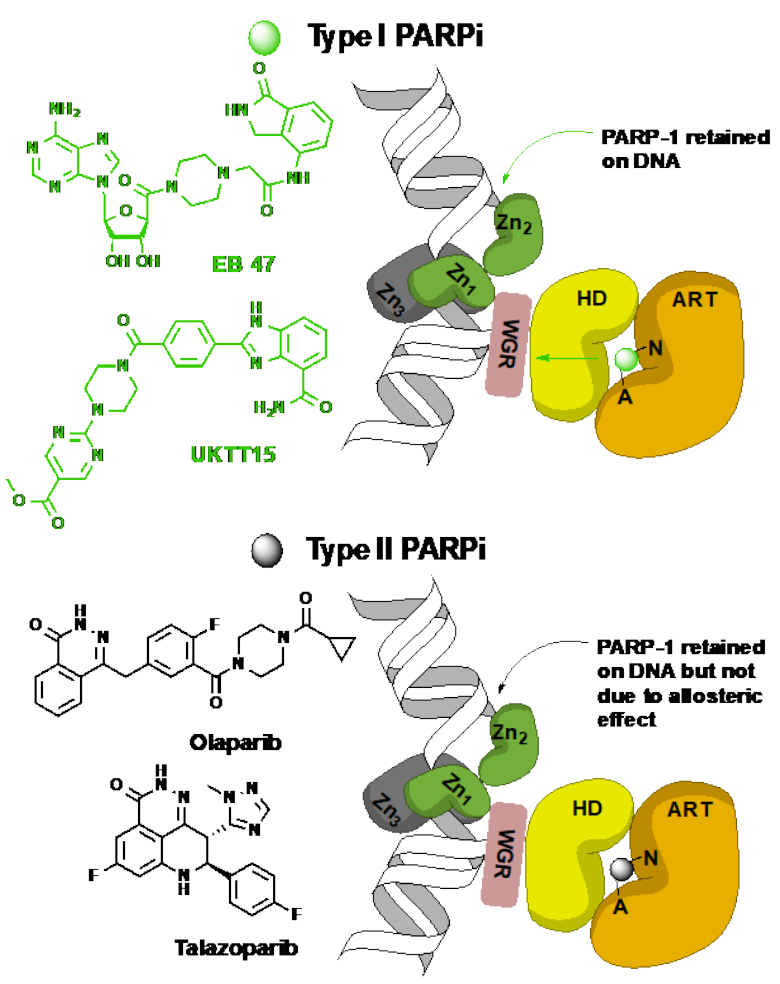

Type II PARPi

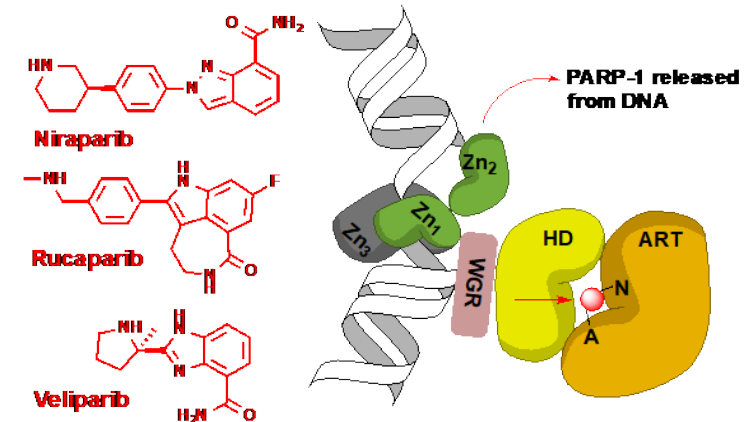

Figur e 2 
A

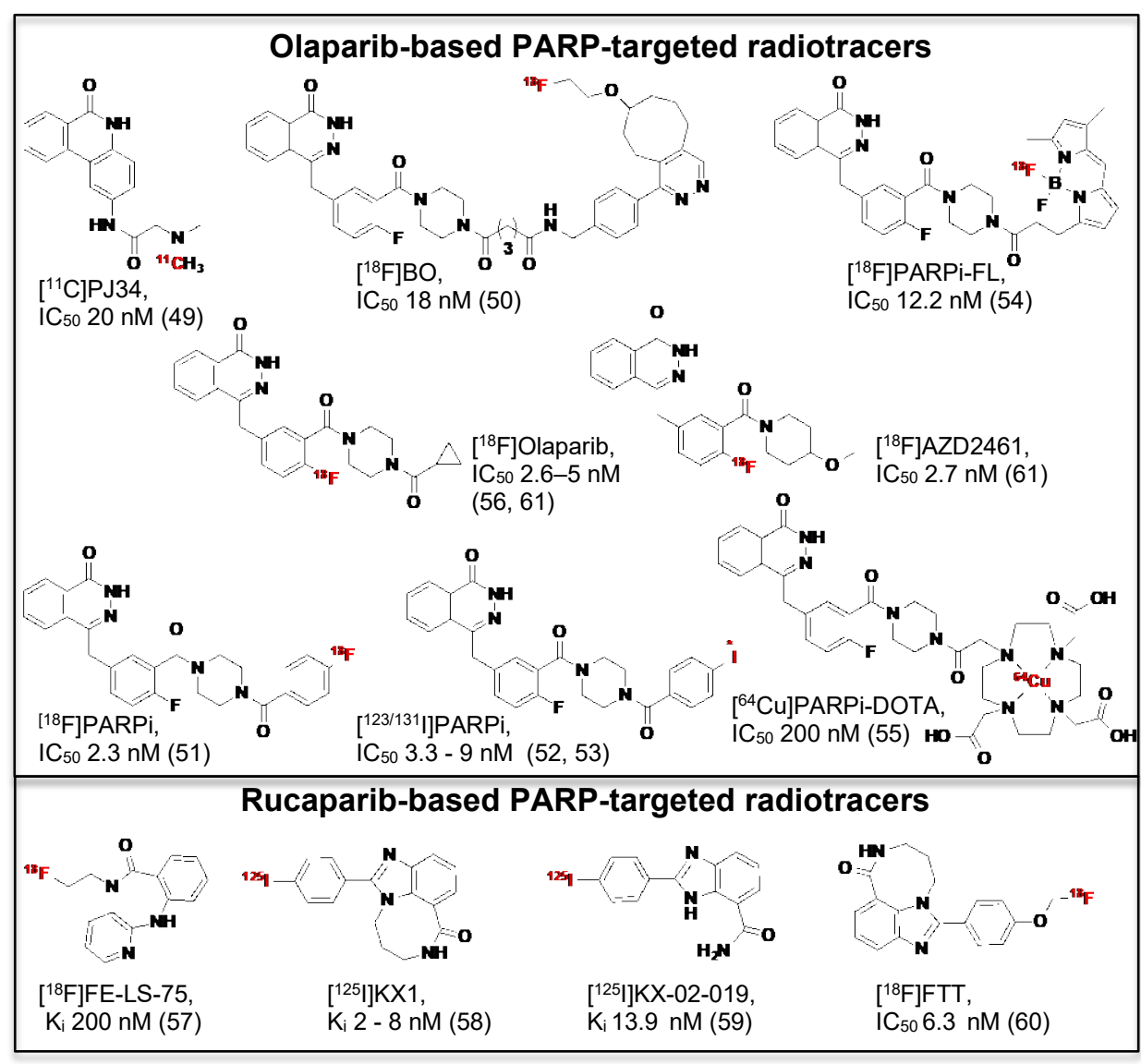

B

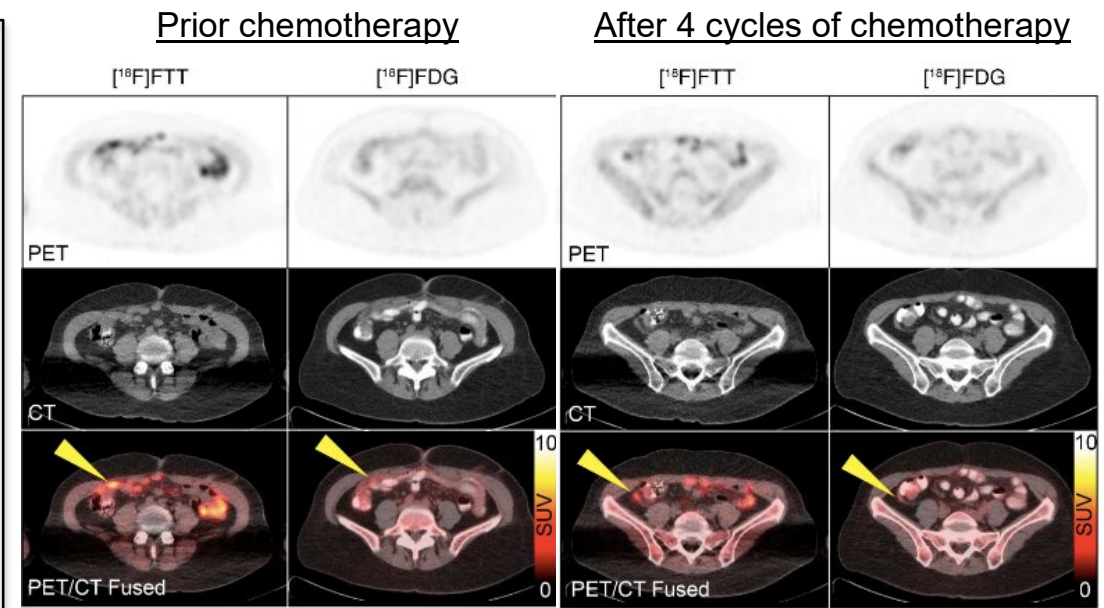

C

${ }^{18} \mathrm{~F}-\mathrm{PARPi}, 30 \mathrm{~min}$

${ }^{18} \mathrm{~F}-\mathrm{PARP} \mathrm{i}, 60 \mathrm{~min}$

18F-PARPi, $120 \mathrm{~min}$

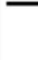

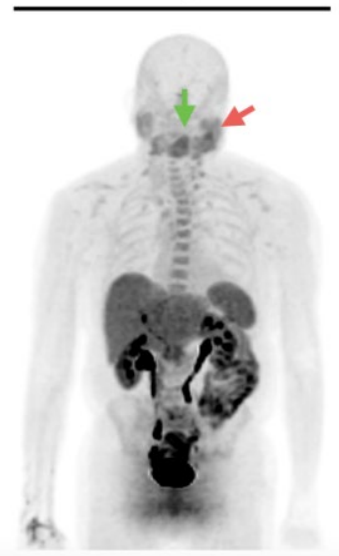


Figure 3:
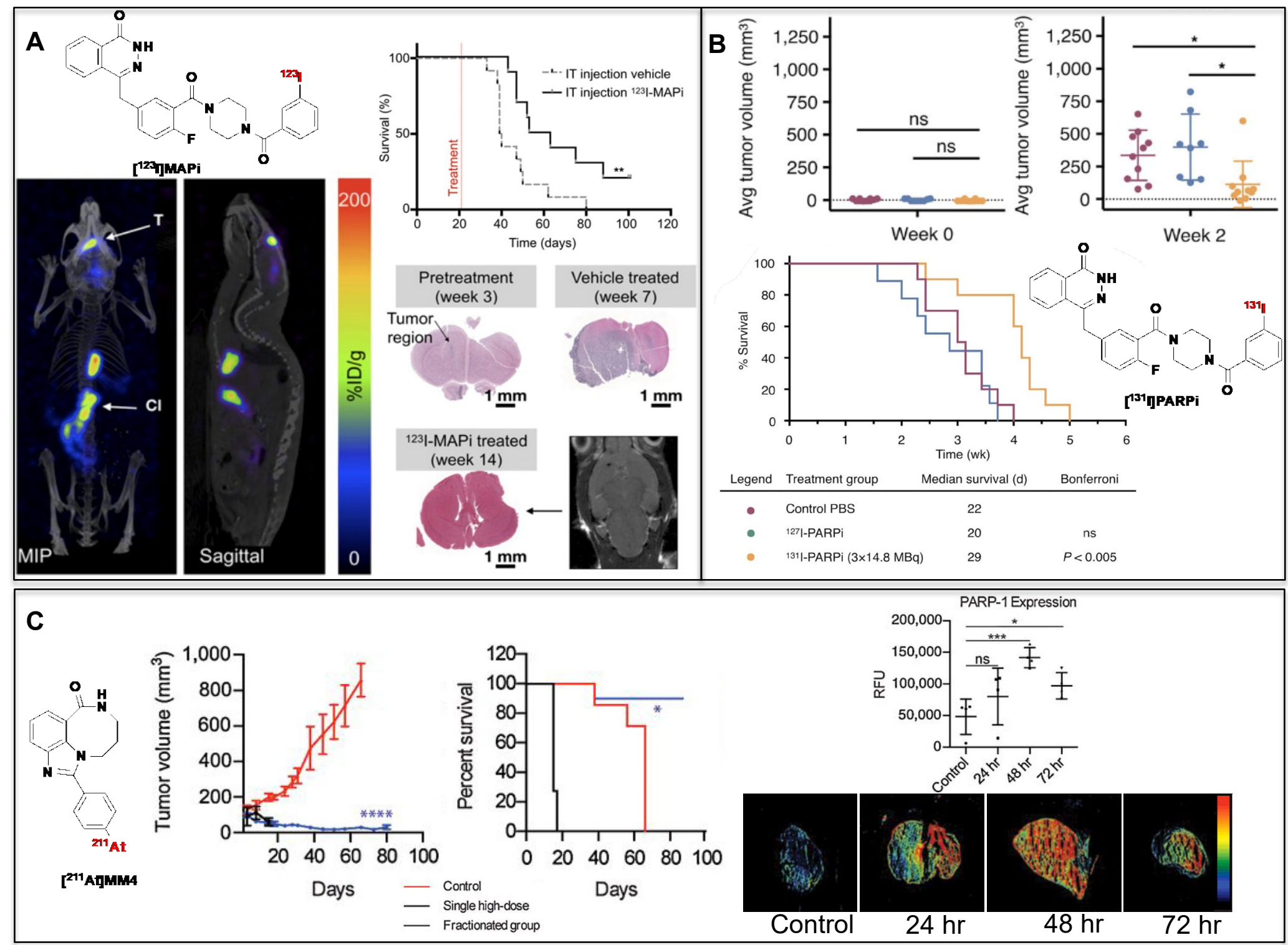
Table 1:

\begin{tabular}{|c|c|c|c|c|c|}
\hline \multicolumn{2}{|c|}{ Combination therapy } & $\begin{array}{l}\text { Additional } \\
\text { Target }\end{array}$ & Treatment & Cancer Type & Publication \\
\hline \multirow{7}{*}{\multicolumn{2}{|c|}{ Genotoxic }} & \multirow{2}{*}{ BCL2 } & Olaparib + Dacarbazine & Uveal Melanoma & (74) \\
\hline & & & Olaparib + Navitoclax & Ewing Sarcoma & $(75)$ \\
\hline & & \multirow{3}{*}{$\mathrm{N} / \mathrm{A}$} & Olaparib + Chloroquine & Ovarian & (76) \\
\hline & & & Talazoparib + TMZ & SCLC & (33) \\
\hline & & & Olaparib + Trabectedin & Ewing Sarcoma & (77) \\
\hline & & \multirow[b]{2}{*}{$\mathrm{N} / \mathrm{A}$} & Olaparib + Cisplatin & Breast & (78) \\
\hline & & & $\begin{array}{c}\text { Niraparib + carboplatin } \\
+ \text { paclitaxel }\end{array}$ & HGSOC & (79) \\
\hline \multirow{4}{*}{\multicolumn{2}{|c|}{ IR }} & \multirow{4}{*}{ N/A } & Olaparib + IR & PDAC & $(80)$ \\
\hline & & & Olaparib + IR & $\begin{array}{l}\text { Renal Cell } \\
\text { Carcinoma }\end{array}$ & $(81)$ \\
\hline & & & Talazoparib + IR & SCLC & (82) \\
\hline & & & Veliparib + IR & GBM & $(83)$ \\
\hline \multirow{17}{*}{ Targeted } & \multirow{12}{*}{ DDR } & ATM & Olaparib + AZD0156 & Breast & (78) \\
\hline & & ATR & $\begin{array}{c}\text { Olaparib + AZD6738 or } \\
\text { MK8776 }\end{array}$ & HGSOC & $(36)$ \\
\hline & & CHK1 & Olaparib + Prexasertib & HGSOC & (37) \\
\hline & & CHK2 & $\begin{array}{c}\text { Talazoparib }+ \\
\text { Prexasertib }\end{array}$ & Gastric & (84) \\
\hline & & CDK2/CDK5 & Olaparib + Dinaciclib & NEPC & (39) \\
\hline & & CDK12 & Veliparib + Dinaciclib & TNBC & $(40)$ \\
\hline & & LMO2 & Olaparib + R-CHOP & DLBCL & $(85)$ \\
\hline & & PIN1 & Olaparib + Tretinoin & TNBC & $(38)$ \\
\hline & & \multirow{4}{*}{ DNA-PK } & Olaparib + AZD7658 & Ovarian & \multirow{4}{*}{ (86) } \\
\hline & & & Olaparib + AZD7659 & TNBC & \\
\hline & & & Olaparib + AZD7660 & NSCLC & \\
\hline & & & Olaparib + AZD7661 & Head and Neck & \\
\hline & \multirow{5}{*}{$\begin{array}{c}\text { Anti- } \\
\text { oncogenic }\end{array}$} & BRD4 & Olaparib + AZD5153 & PDAC & (41) \\
\hline & & BRD5 & Olaparib + JQ1 & PDAC & $(42)$ \\
\hline & & BRD6 & Talazoparib + JQ1 & Breast & $(41)$ \\
\hline & & MYCN & Olaparib + Danusertib & NEPC & (87) \\
\hline & & AR & $\begin{array}{c}\text { Olaparib }+ \\
\text { enzalutamide }\end{array}$ & CRPC & $(43)$ \\
\hline
\end{tabular}


Table 2

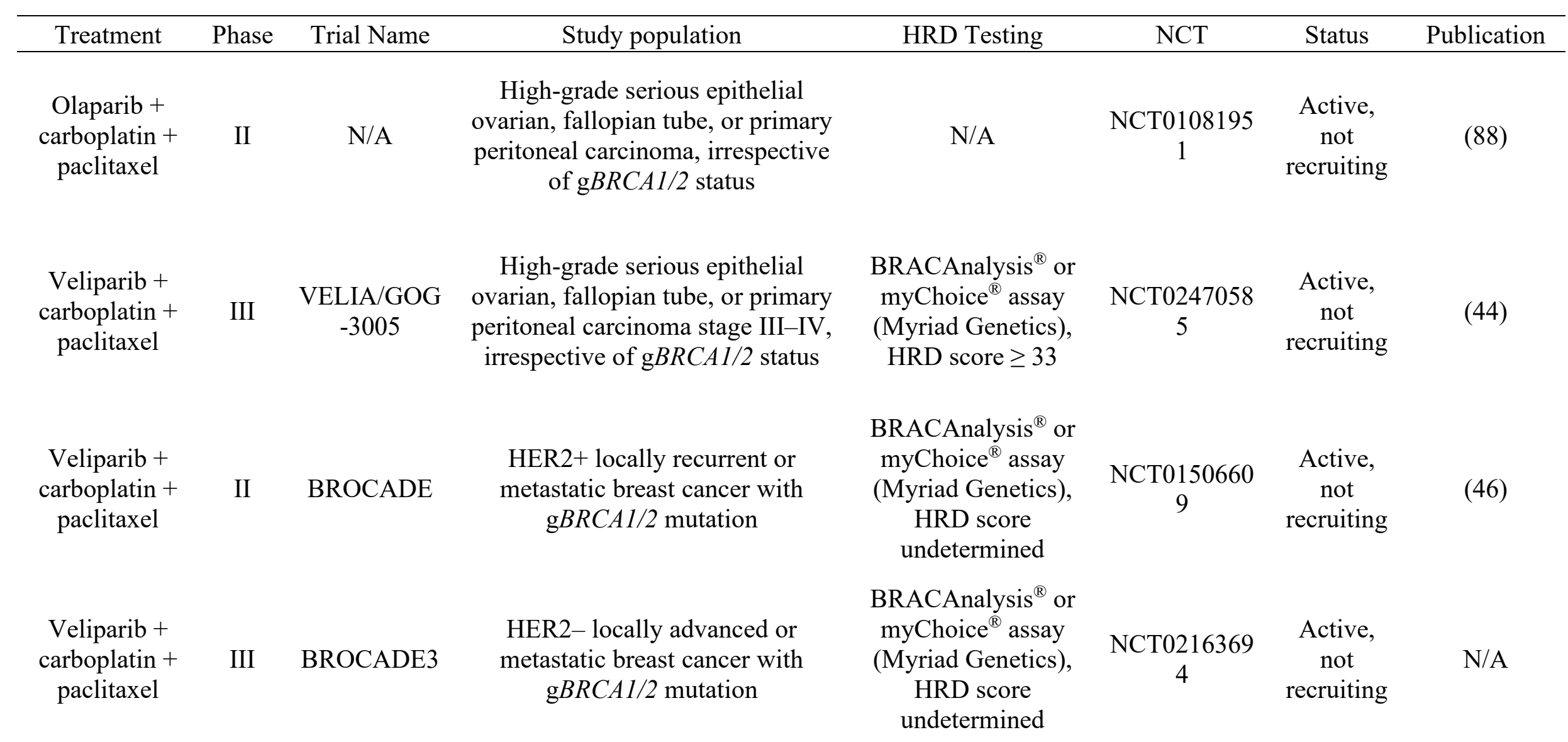


Veliparib +
carboplatin +

paclitaxel

Veliparib +

TMZ

Veliparib + cisplatin + etoposide

Veliparib + cisplatin + gemcitabine

Veliparib + FOLFIRI
TNBC stage II-III (T1N1-2 or T2-4N0-2), irrespective of $\mathrm{g} B R C A 1 / 2$ status

SCLC sensitive or refractory to platinum-based chemotherapy, irrespective of $\mathrm{g} B R C A 1 / 2$ status

SCLC with presence of extrathoracic metastatic disease, malignant pleural effusion, and bilateral or contralateral supraclavicular adenopathy, irrespective of $\mathrm{g} B R C A 1 / 2$ status

Locally advanced and metastatic PDAC stage III-IV, with $\mathrm{g} B R C A 1 / 2$ and PALB2 mutation

Locally advanced and metastatic PDAC stage IV, irrespective of $\mathrm{g} B R C A 1 / 2$ status
BRACAnalysis $^{\circledR}$ assay (Myriad

Genetics), HRD

NCT0203227

Active,

7

not

score undetermined

recruiting

$$
\text { N/A } \quad \begin{array}{cc}
\text { NCT0163854 } \\
6
\end{array}
$$

BRACAnalysis ${ }^{\circledR}$ or myChoice ${ }^{\circledR}$ assay (Myriad Genetics), HRD score undetermined

$\begin{array}{cc}\text { NCT0158580 } & \text { Active, } \\ 5 & \text { not } \\ & \text { recruiting }\end{array}$

N/A

NCT0289035
5

Active,

not

recruiting 
High-grade serious epithelial Olaparib + Bevacizumab

PAOLA-1 Cediranib

I/II

N/A

Olaparib +

Abiraterone

II N/A

$$
\begin{array}{r}
\text { mCRPC, irrespective } \\
\text { status }
\end{array}
$$

High-grade serious epithelial ovarian, fallopian tube, or primary peritoneal carcinoma and TNBC, with $\mathrm{g} B R C A 1 / 2$ mutation eal carcinoma, irrespective of $\mathrm{g} B R C A 1 / 2$ status

Olaparib + Durvalumab + Bevacizumab

Olaparib + Durvalumab + Bevacizumab Advanced or metastatic solid tumor, irrespective of $\mathrm{g} B R C A 1 / 2$ status lung, prostate and colorectal cancers with $\mathrm{g} B R C A 1 / 2$ mutation
myChoice ${ }^{\circledR}$ assay (Myriad Genetics), HRD score $\geq 42$

\section{BRACAnalysis ${ }^{\circledR}$} assay (Myriad

Genetics), HRD score undetermined

$\begin{array}{cc}\text { NCT0111664 } & \text { Active, } \\ 8 & \text { not } \\ & \text { recruiting }\end{array}$

$$
\begin{array}{cc}
\text { NCT0197221 } & \text { Active, } \\
7 & \text { not } \\
& \text { recruiting }
\end{array}
$$

BRACAnalysis ${ }^{\circledR}$ or myChoice ${ }^{\circledR}$ assay

(Myriad Genetics), HRD score undetermined

$\begin{array}{cc}\text { NCT0273400 } & \text { Active, } \\ 4 & \text { not } \\ & \text { recruiting }\end{array}$

BRACAnalysis ${ }^{\circledR}$ or myChoice ${ }^{\circledR}$ assay (Myriad Genetics), HRD score 